\title{
Globus Pallidus
}

National Cancer Institute

\section{Source}

National Cancer Institute. Globus Pallidus. NCI Thesaurus. Code C12449.

A sub-cortical, paired nuclei that, though migrated to the telencephalon, is a critical part of the basal ganglia, along with the caudate nucleus and the putamen, the latter of which it joins to form the lentiform nucleus. Each nucleus is subdivided into internal and external parts, called globus pallidus interna and globus pallidus externa, which are separated by an internal medullary lamina. 